\title{
Research Paper \\ Prediction of Psychological Well-Being of the Elderly Based on the Power of Stress Management and Social Support
}

\author{
Abdollah Moatamedy ${ }^{1}$, Ahmad Borjali ${ }^{1},{ }^{*}$ Maryam Sadeqpur ${ }^{1}$
}

1. Department of Psychology, Faculty of Psychology \& Education, Allameh Tabataba'i University, Tehran, Iran.

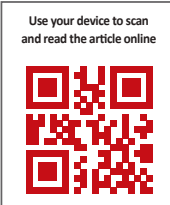

Cltration: Moatamedy A, Borjali A, Sadeqpur M. [Prediction of Psychological Well-Being of the Elderly Based on the Power of Stress Management and Social Support (Persian)]. Iranian Journal of Ageing. 2018; 13(1):98-109. https://doi.org/10.21859/sija.13.1.98

Received: 28 Sep. 2017 Accepted: 11 Feb. 2018

Key words:

Psychological wellbeing, Elderly, Social support, Stress management ability

\begin{abstract}
A B S T R A C T
Objectives Owing to the fact that interpersonal bonds of social support can improve health under stressful situations, social support can have a protective and moderating role against the harmful effects of stress. The present research aims to predict the relationship between psychological well-being and the ability to manage stress and social support.

Methods \& Materials This descriptive-analytic research is correlational and is done through the sectional method. The society includes all the elderly living in Tehran (60 years and older), and the sample was selected among the old people who appeared in parks during May and June, 2016 in district 8 in Tehran. The sample size is determined using Cochran formula ( $n=131$ people). In this research, the short form of Rif psychological well-being scale (1989), Kalzbyk stress management scale (1990), and Philips social support scale (1977) have been applied. The data analysis was performed through multiple regressions.

Results The results show that social support and stress management with $t=2.23$ and $t=4.79$, respectively, on level Beta $=0.05$ will predict psychological well-being.

Conclusion The results showed that social support and stress management variables can predict psychological well-being variable among the elderly. Therefore, the use of active coping styles is recommended.
\end{abstract}

\section{Extended Abstract}

\section{Objectives}

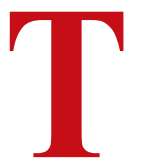

he increasing population of the elderly worldwide and also in Iran reveals the need for conducting research in this regard. However, most of the studies conducted in Iran in the field of aging are new and limited and focus on the physical health of the elderly people. It should be noted that elderly studies will have a growing value and credibility for different policies including studies on the physical and mental health of the elderly people. Psychological well-being affects disease control and the reactivity of people when facing problems. Interpersonal links of social support can promote health. Under stressful circumstances, social support plays the role of a moderator and protector from the harmful effects of stress. The current study was conducted with the aim of predicting the relationship between psychological well-being and stress management ability and social support. 


\section{Methods and Materials}

This descriptive-analytic study is a cross-sectional correlational research conducted in May and June, 2017. Statistical population of this study included all elderly people living in Tehran (60 years and older). The subjects were selected from among the elderly in parks (Parks of Haft Howz, Fadak, Taslihat, Bahares$\tan$ ) in district 8 of Tehran province using the available sampling method. Using the Cochran formula, the sample size was determined to be 131. In this study, a short form (18 questions) of the Reef Psychological Well-being Scale (1989) was used to measure the psychological well-being of the elderly. The Calzbick Stress Management Scale (1990) was employed to assess the stress management of the elderly, and the Social Support Scale of Philips (1977) was used to measure social support of the studied elderly. In the present study, questionnaire method was used individually to collect information.

An inclusion criterion to participate in this study was the ability to read and write. The data collected from the questionnaires were analyzed using SPSS21 software and simultaneous and simple multivariate regression testing. This study was approved in terms of ethics of conducting research by the professors of Faculty of Psychology and Educational Sciences at Allameh Tabatabai University of Tehran. In this study, all moral considerations including the informed consent and the confidentiality of the information were observed. The purpose of the study was explained individually to the elderly subjects, and their permissions were obtained for performing the study and using their information. The elderly under study were free to accept or refuse to participate in the research. As a result, a number of participants agreed to cooperate at the beginning but refused to complete the questionnaire and left the research, and some other subjects replaced them. The participants were assured that the gathered information will be anonymous and that confidentially will be maintained until the end of the study. All the scientific materials are presented with reference to their source.

\section{Results}

The number of male and female participants was equal in terms of demographic characteristics. A total of 65 men and 65 women, equivalent to $50 \%$ of both genders, participated in this study. Of them, $87 \%$ of the elderly were married and the remaining $13 \%$ were single. With regards to age, $85 \%$ of the participants were 60 and 75 years of age and $11 \%$ were more than 75 years of age. In terms of education, $36 \%$ of the elderly had an elementary degree, $28 \%$ had a secondary degree, $24 \%$ had a diploma, and $12 \%$ had a university degree. On the scale of psychological well-being, getting higher score meant better psychological well-being. Correlation of the short version of Reef Psychological Well-being Scale with the original scale fluctuated between of 0.7 to 0.89 . In the present study, scale validity using Cronbach's Alpha was 0.65 .

In the scale of coping with the stressful conditions by Calzbic, the scoring was based on 5-point Likert scale, where the maximum value for each item was 5 and the least one was 1 . The range of changes included three types of confrontational behaviors in a way that the score for each of the triple confrontational behaviors, i.e. problem-oriented, emotion-oriented, and avoidance-oriented, was from 16 to 80 . In other words, the dominant confrontational behaviors of the individual were determined according to the score obtained in the test. In other words, a behavior with a higher score was considered as confrontational behavior for the individual. Boysan (2012) has reported the alpha coefficient of sub-scales as follows: Problem-oriented coping was 0.72 , emotion-oriented coping was 0.77 , and avoidance-oriented coping was 0.74 . In the present study, alpha coefficient of problem-oriented coping was 0.75 , emotion-oriented coping was 0.72 , and avoidance-oriented coping was 0.57 . On the Phillips Social Support Scale, getting a high score represents higher social support. In the current study, Cronbach's alpha for the whole questionnaire was obtained to be 0.70. Simultaneous regression was used to answer the assumption that "stress management ability and social

Table 1. Fitting result of the regression model

\begin{tabular}{|c|c|c|c|c|c|}
\hline \multirow{2}{*}{ Model } & \multicolumn{2}{|c|}{ Non-Standard Coefficients } & \multirow{2}{*}{$\begin{array}{c}\text { Standard Coefficients } \\
\text { Beta Value }\end{array}$} & \multirow{2}{*}{ T Value } & \multirow{2}{*}{ P Value } \\
\hline & B Value & Standard Error & & & \\
\hline Constant value & 38.419 & 5.535 & & 6.941 & 0.001 \\
\hline Social support & 0.520 & 0.232 & 0.181 & 2.236 & 0.27 \\
\hline Stress management ability & 0.409 & 0.085 & 0.388 & 4.798 & 0.001 \\
\hline
\end{tabular}


support can predict psychological well-being in the elderly under study".

$R$ value or correlation between predictor and dependent variables equaled to 0.46 , and the value of $\mathrm{R}^{2}$ equaled to 0.21 . That is, the amount of variance that the predictor variables of social support and stress management explain for dependent variable, i.e. psychological well-being, equaled to 0.21 . Using the variance analysis of the obtained $\mathrm{F}$ value, the significance of regression model equaled to 17.54. This amount is significant at the level of 0.01 .

Table 1 shows the regression coefficients related to predictor variables of social support and stress management. Beta coefficient value for social support equaled to 0.18 , which is significant at the level of 0.05 . This coefficient, in fact, shows the magnitude of the impact of a predictive variable on the dependent variable that is not high. The beta coefficient value for stress management variables equaled to 0.38 , which is significant at the level of 0.01 . Considering the amount of beta coefficients for two predictor variables, it can be said that stress management variable has more relationship with the dependent variable, i.e. psychological well-being. The results show that social support with $\mathrm{t}=2.23$ at the level of $\alpha=0.05$ and stress management with $\mathrm{t}=4.79$ at the level of $\alpha=0.01$ predict psychological well-being.

\section{Conclusion}

The present study was conducted with the aim of predicting the relationship of psychological well-being with stress management ability and social support. The findings resulting from the hypothesis of stress management ability and social support confirmed the relationship of stress management and social support with psychological well-being in the elderly under study. This indicates that at least one of the predictor variables (stress management or social support) was effective in criterion variable (psychological well-being). The review of the literature has confirmed the relationship between the research variables. Social support as a facilitator of stress compliance is considered as one of the sources of confrontation that affects the promotion of health in older people. Having friends helps people to consider stressful events less threatening and controllable and reduce negative feelings resulting from stress. Therefore, these helpful interventions are recommended for the elderly to acquire and maintain effective resources and coping skills. The use of stress management practices, which includes efforts to minimize, avoid, tolerate, and accept stressful conditions, helps to balance physical and mental health and promote successful aging process. Thus, active coping styles are recommended.

\section{Acknowledgments}

Mr. Mehdi Nezhad has financially supported this article.

\section{Conflict of Interest}

The authors declared no conflicts of interest. 


\title{
ييشبينى بهزيستى روانشناختى سالمندان براساس توان مديريت استرس و حمايت اجتماعى
}

\author{
عبدالله معتمدى'، احمد برجعلى'؛ "مريم صادقيور' \\ ا- كروه روانشناسى، دانشكده روانشئاسى و علوم تربيتى، دانشكاه علامه طباطبائى، تهران، ايران.
}

\begin{abstract}
حكSد

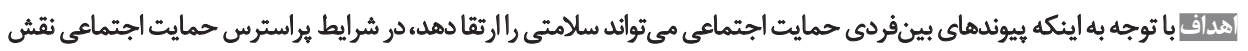

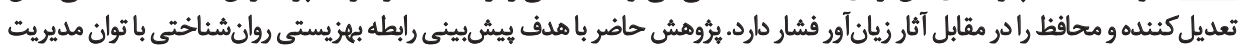
استرس و حمايث اجتماعى انجام شدائ.

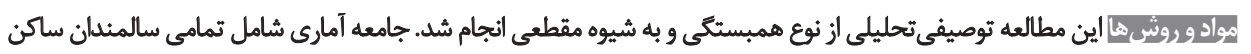

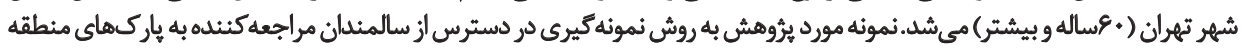

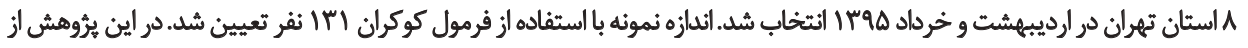

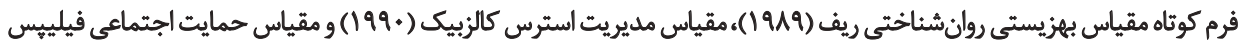

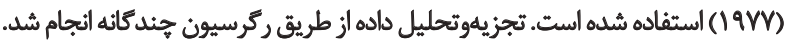

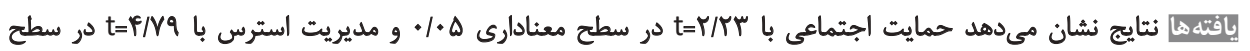

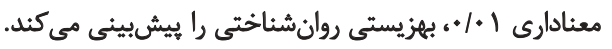

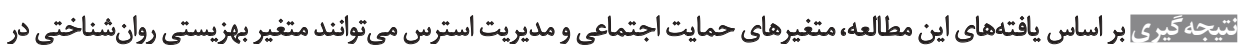

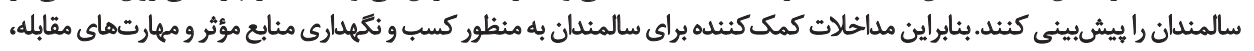

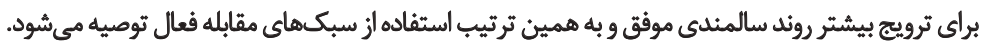

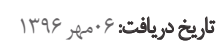

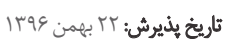

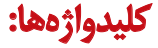
بهزيستي روانشيناختي، سالمندان، حمايت روانشين اجتماعي، توان مديريت المايت استرس

باوجود رشد جمعيت سالمند هنوز بر نيازهاى سالمندان به عنوان كروه آسيبيذير جامعاده تمركز نشده است.

مقدمه

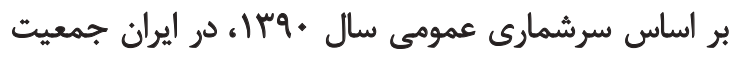

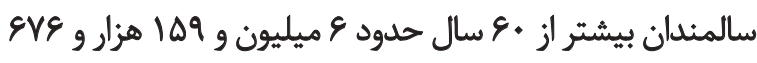

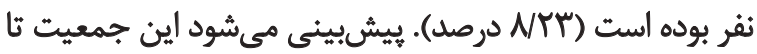

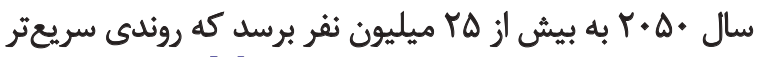

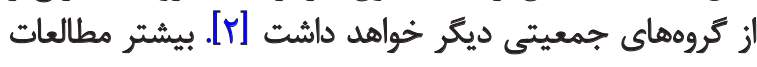

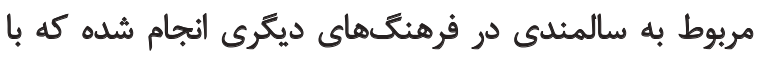

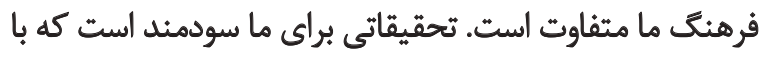

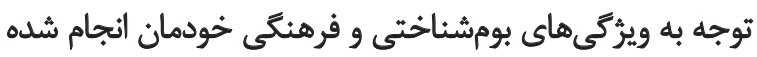

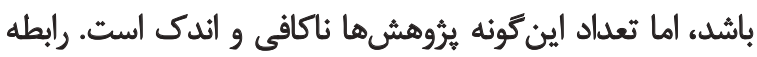

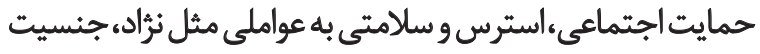

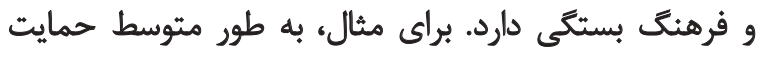

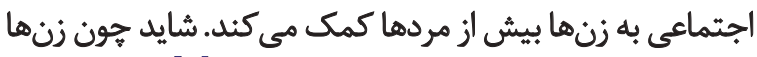

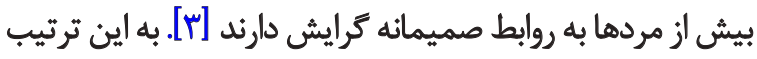

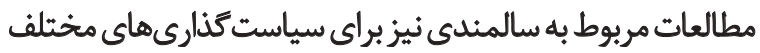

بيشتر مردم تمايل دارند عمر طولانى داشته باشند، اما از دازئن

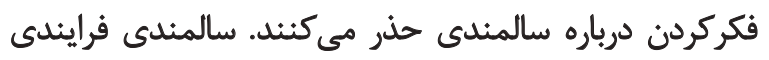

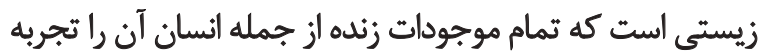

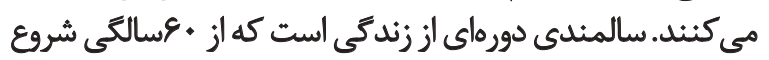

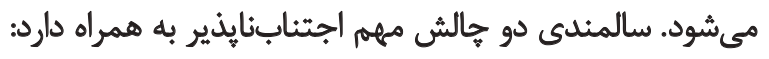

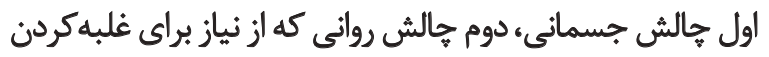

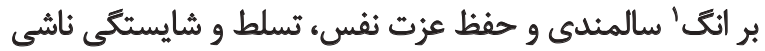
مىشود [1]. افرزايش جشمعير جمعيت سالمندان در جهان و ايران اهميت

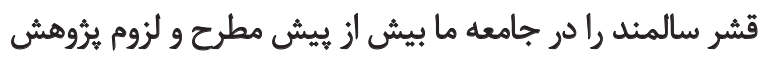

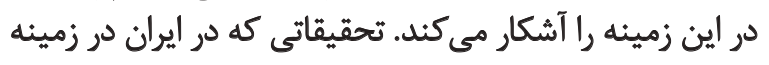

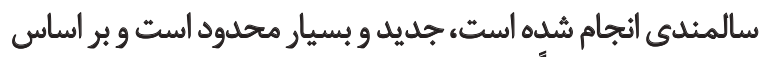
آن، سالمندى غالباً در مفهوم سلامت فيزيكي بردي بررسى شده است. 1. Stigma

مريم : نويسنده مسئول: مريم صادقيور نشائى: تهران، دانشعاه علامه طباطبائي، دانشكده روانشئاسى و علوم تربيتى، كروه روانشناسى.

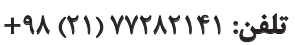
يست الكترونيكي: sadeqpur931@atu.ac.ir 
خارجىاى براى مقابله باعوامل استرسزاست [V]] درباره سبكى كه در آن حمايت اجتماعى به كنترل فشار روانى

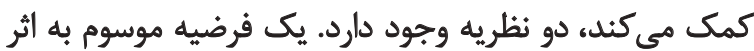

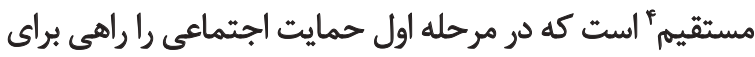

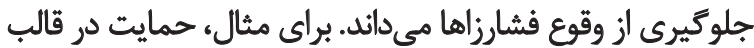

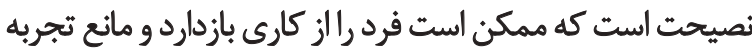

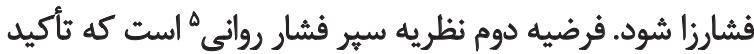

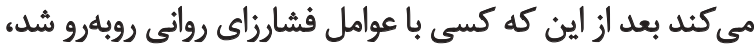

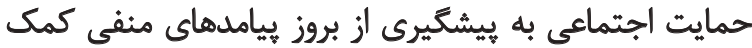

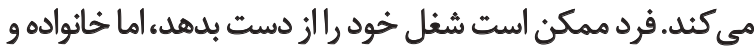

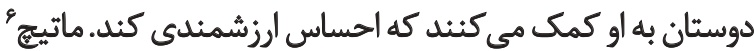

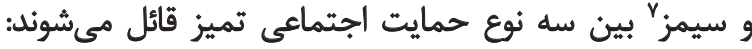

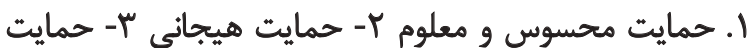

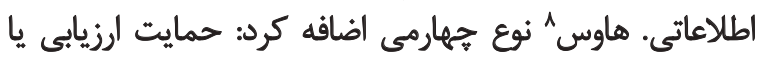
خودسنجي [A، هدف از دستاور دهاى علمى بشر فقط افزايش اميد به زندكى در

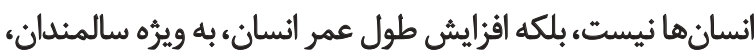

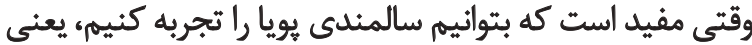

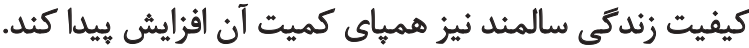

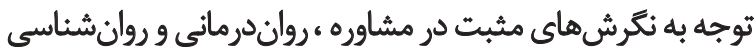

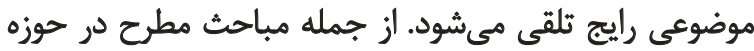

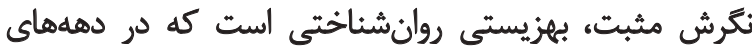

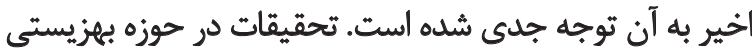

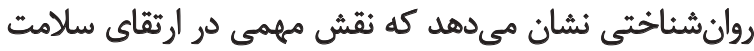

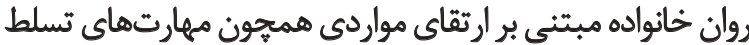

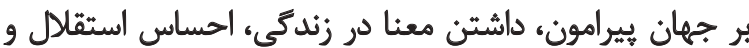

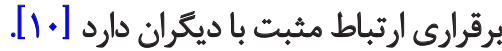

شمارى از مطالعات نشان مىدهد كه حمايت اجتماعى با

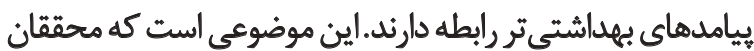

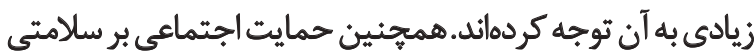

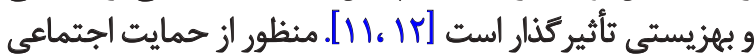

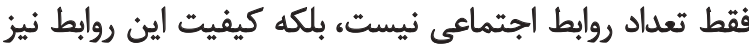

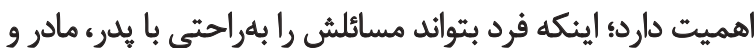

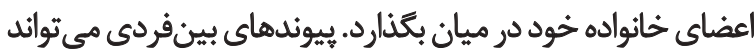

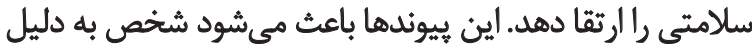

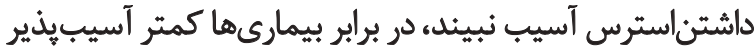

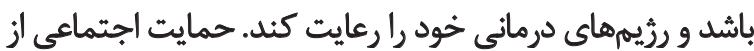

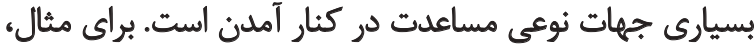
4. Direct effect theory
5. Stress buffering theory
6. Matich
7. Sims
8. House

از جمله مطالعات مربوط به سلامت جسمى و رواني سالمندان

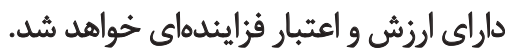
منظور از بهزيستى در روانشناسى مثبت مرا افزايش ميزان

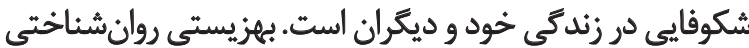

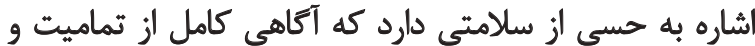

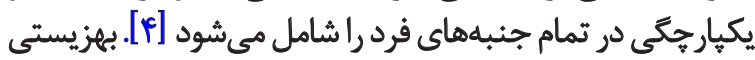

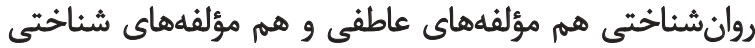

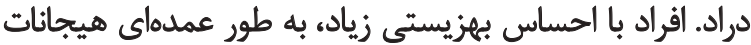

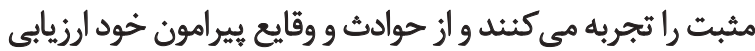

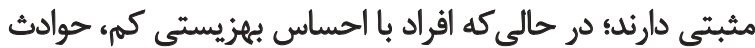

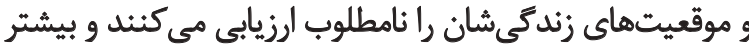

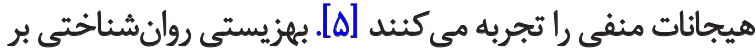

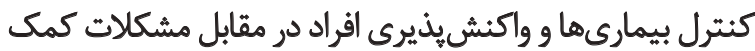

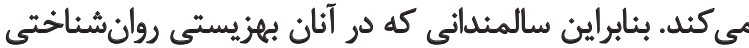

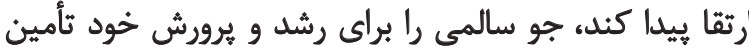

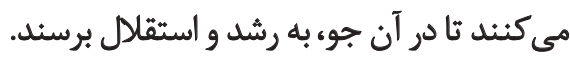

كارل ريفَ شش مؤلفه براى بهزيستى روانشناختى توضيح

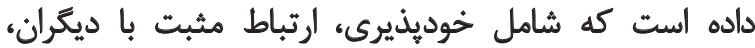

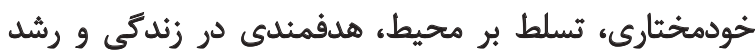

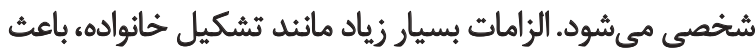

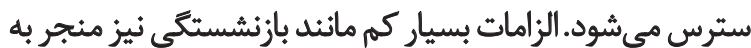

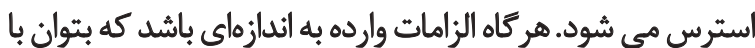

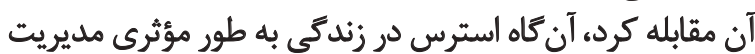

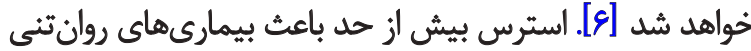

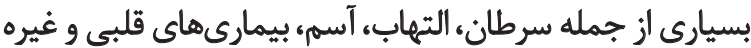

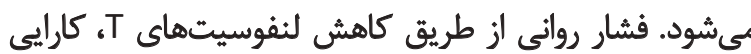

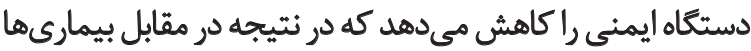

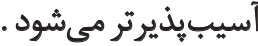

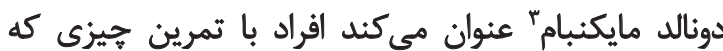

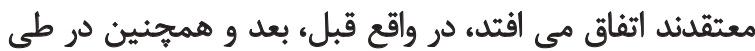

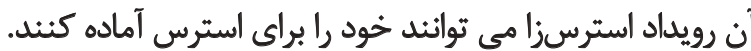

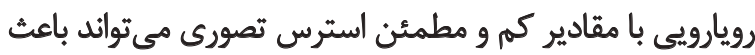

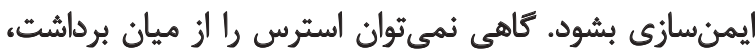

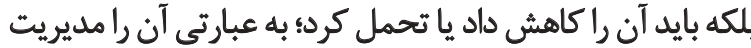

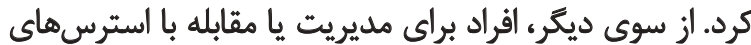

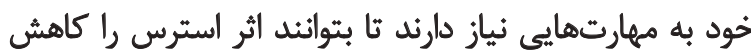

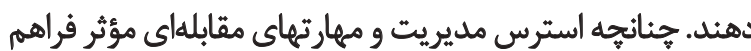

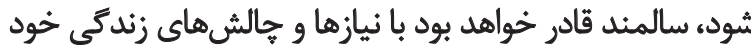

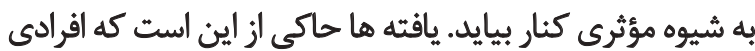

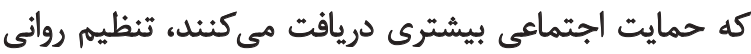

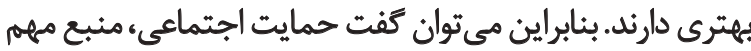

3. Michenbaum Donald 
يويشنهاد مي كند [Yr]

حمايت اجتماعى علاوه بر اينكه به افزايش رضايت از زندگى بائى

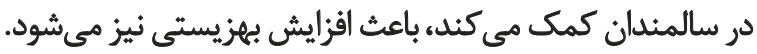

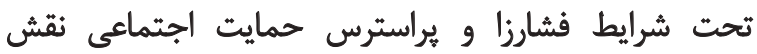

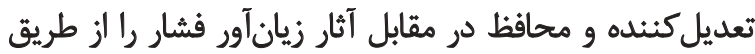

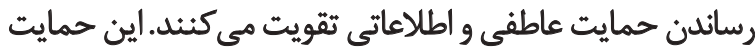

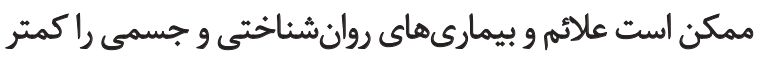

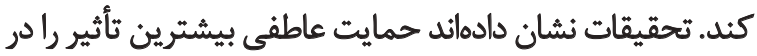

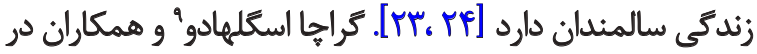

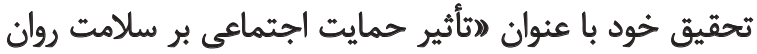

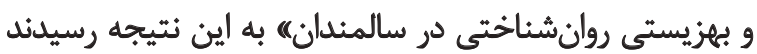

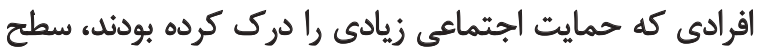

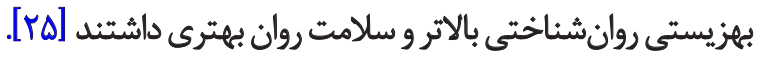

يكسين جن و توماس هوك فلى " در مطالعهاى با عنوان

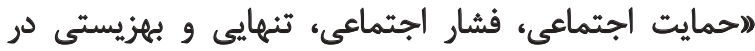

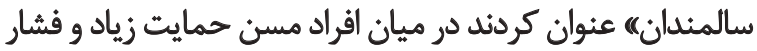

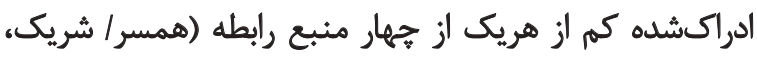

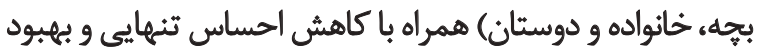

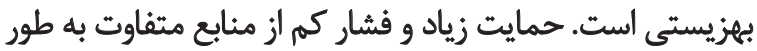

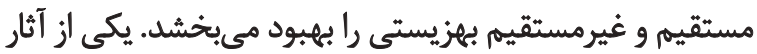

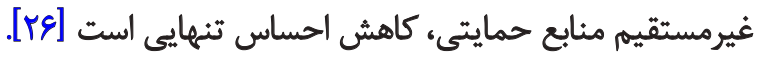

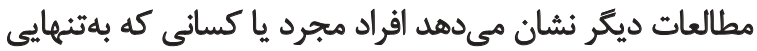

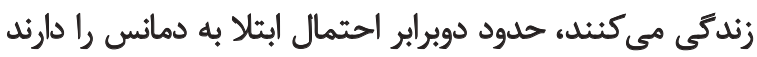

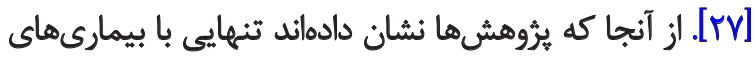

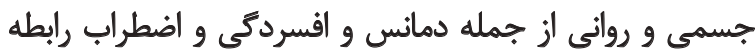

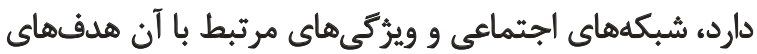
جذابى براى مداخلات بالقوه هستئد.

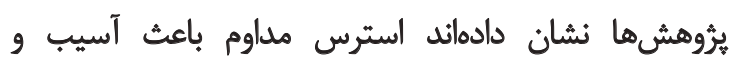

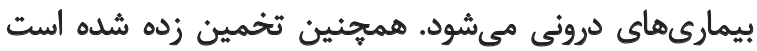

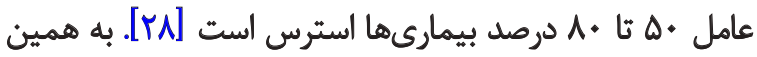

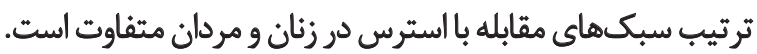

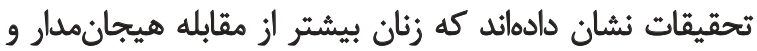

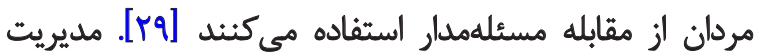

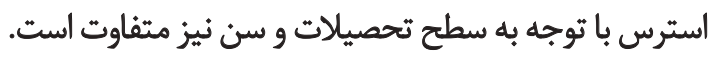

در يروهش ديكرى سهم منابع مقابله والكوهاى مقابله با استرس

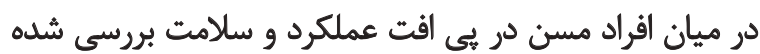

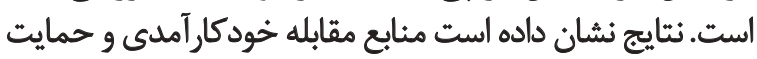

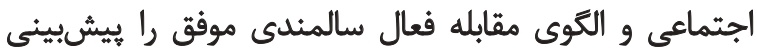

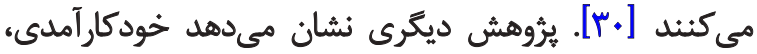

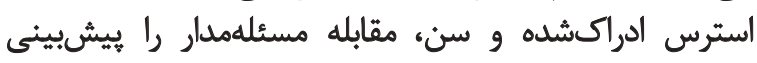

9. Graca Esgalhado

10. Yixin Chen, Thomas Hugh Feely

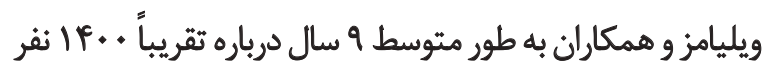

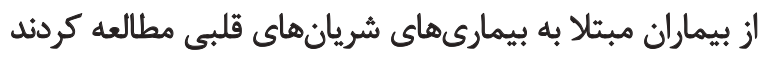

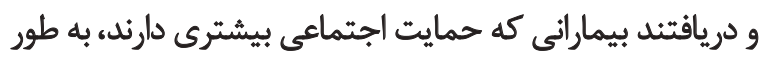

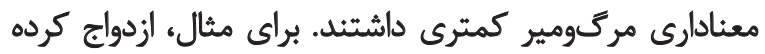

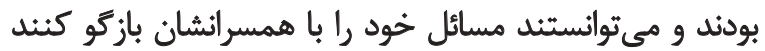

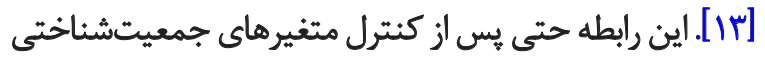

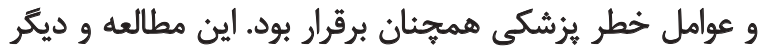

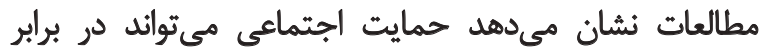
بيامدهاى ناكوار بهداشتى نقش حائل را داشته باشد.

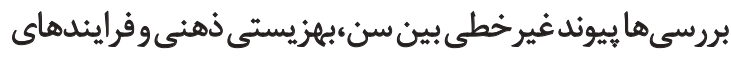

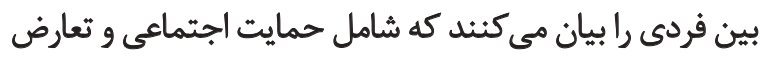

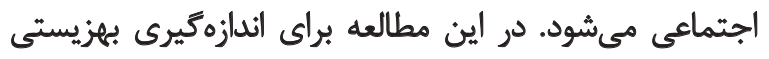

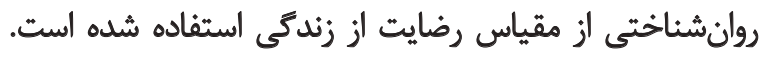

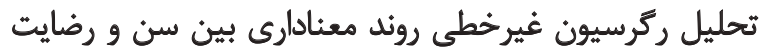

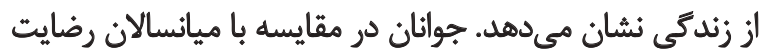

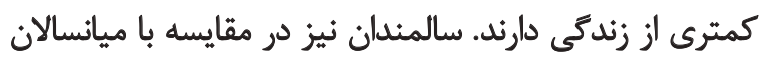

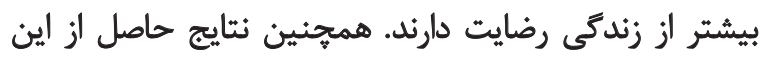

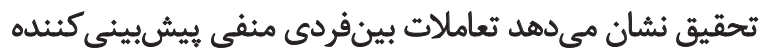

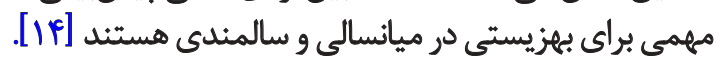
تفاوتهاى مرثبط با سن با بهزيستى روانى بهخوبى مستئن

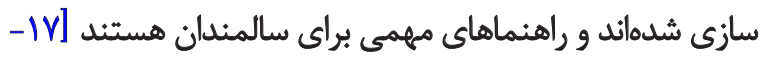

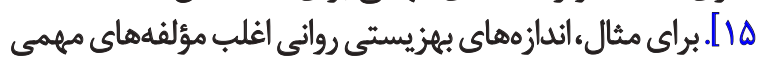

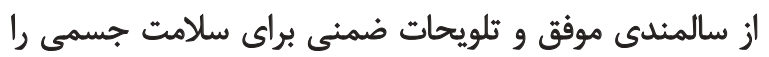

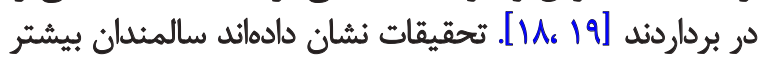

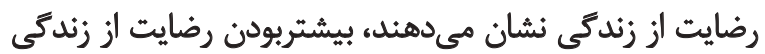

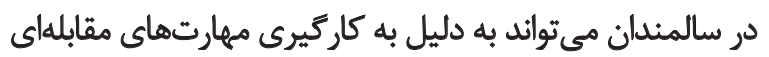

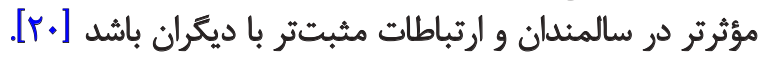

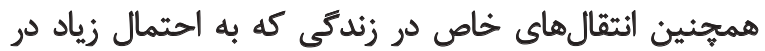

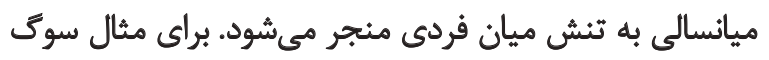

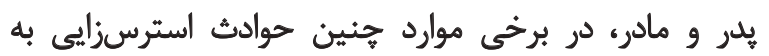

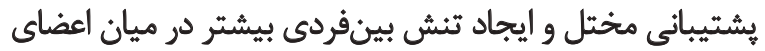

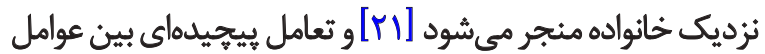
اجتماعى خانواده به وجود مى آيد.

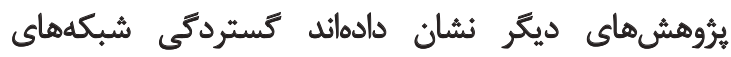

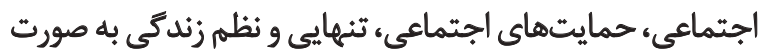

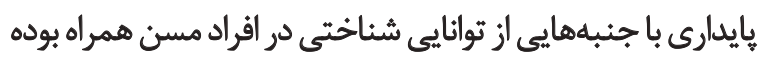

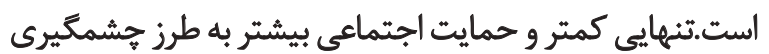

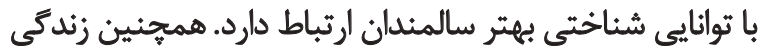

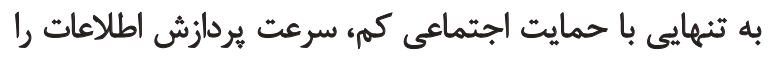

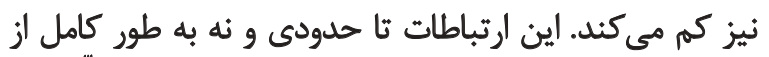

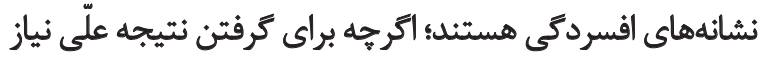

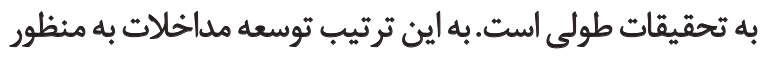

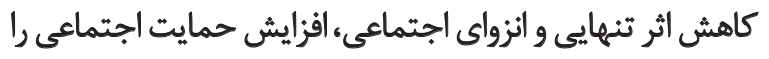


براى تكتك سالمندان مذكور توضيح داده شد و از ايشان

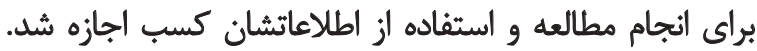

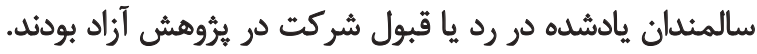

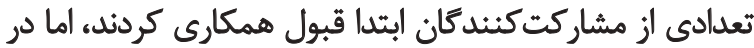

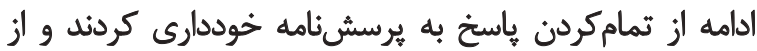

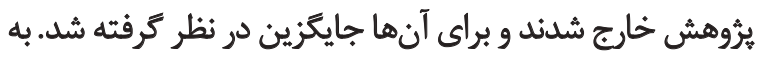

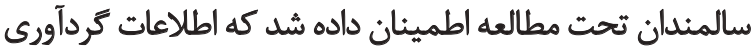

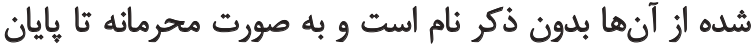

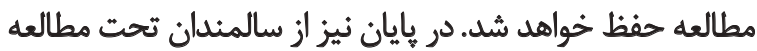

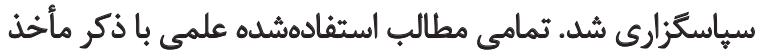
آنها الرائه شده است.

در اين ثروهش از فرم كوتاه (A) سؤالى) مقياس بهزيستى

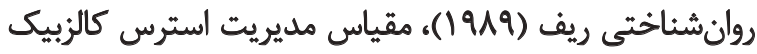
(1999•) و مقياس حمايت اجتماعى فيلييس (19VV) استفاده (199)

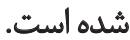

مقياس بهزيستى روانشناختى ويف" (149) در اين بثروهش از نسخه كوثاه (N/اسؤالى) مقياس بهزيستى

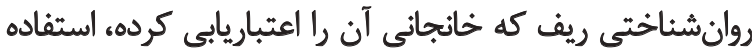

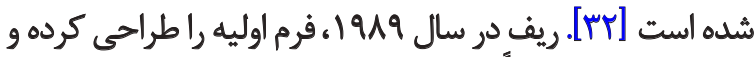

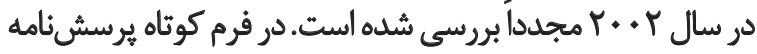

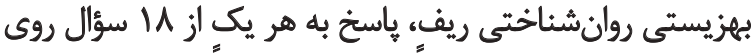

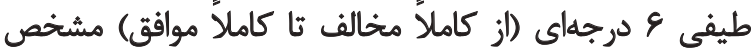

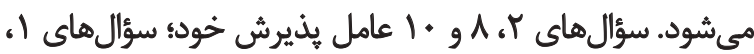

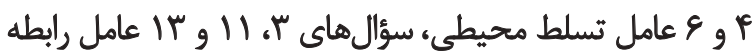

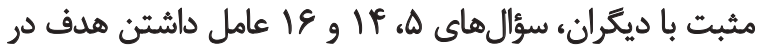

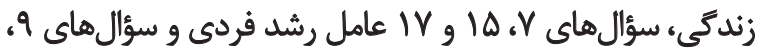

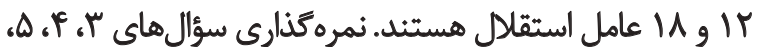

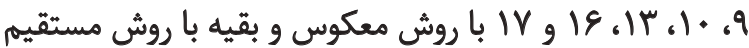

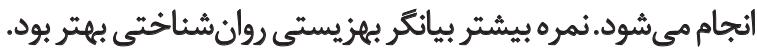

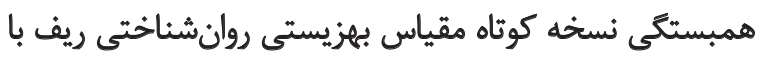

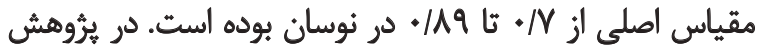

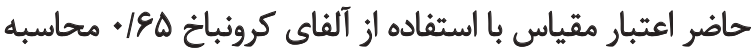

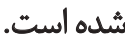

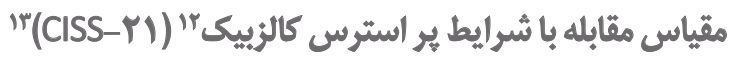

در يثروهش حاضر از فرم كوتاه مقياس خودسنجى مقابله با بالي

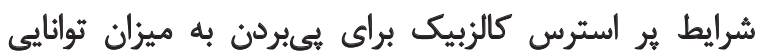

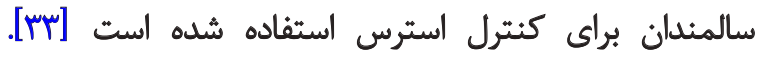

11. Ryff Scale Psychological Well-Being (RSPWB)

12. Calsbeek

13. Shortened version of the coping Inventory for stress full situations
مي كنئد. همجنين نتايج ثأييد كردند سبك مقابله هيجان مدار باريا

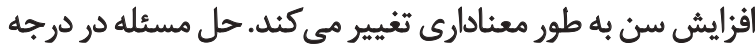

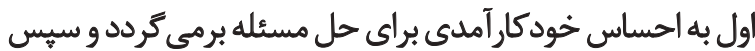

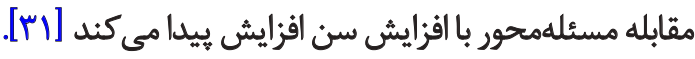

سالمندى زمان آسيبيذيرى خاص براى بيمارهاى مزمن

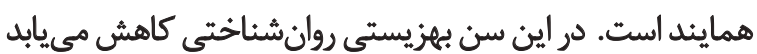

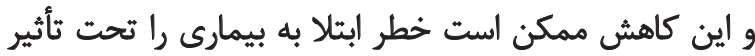

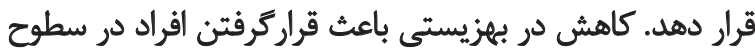

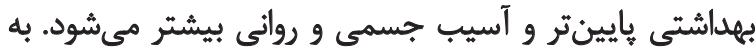

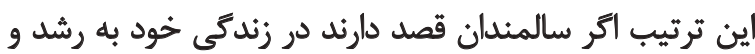

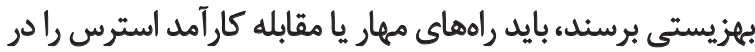

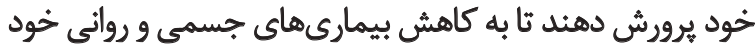

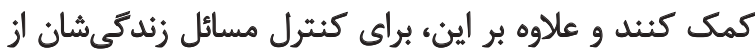

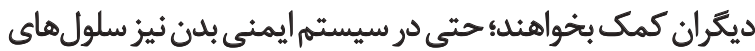

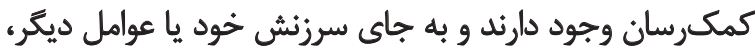

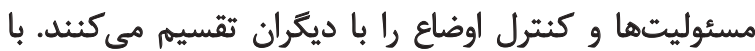

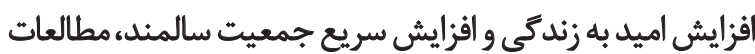

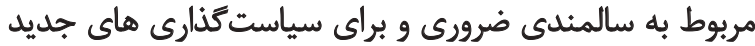
اقتصادى، حمايتى و سلامت جسم و روان لازم است برى سياست

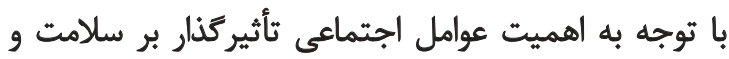

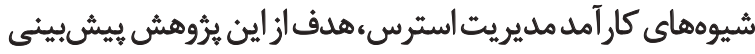

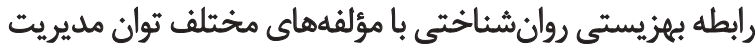

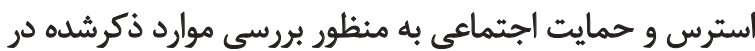

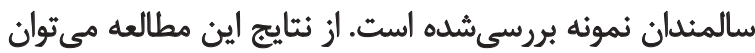

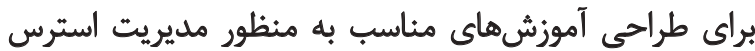

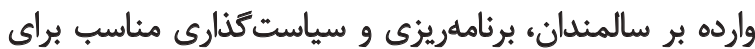

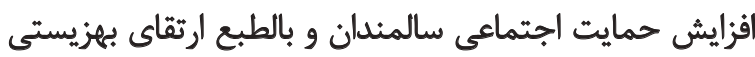
روانشناختى سالمندان استفاده كرد.

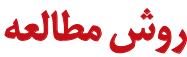

اين مطالعه توصيفىتحليلى از نوع همبستىى و به شيوه

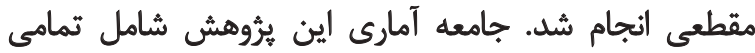

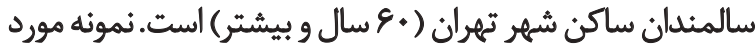

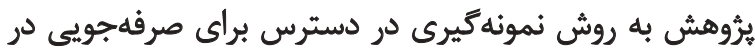

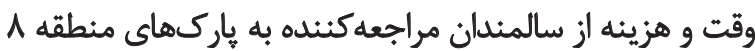

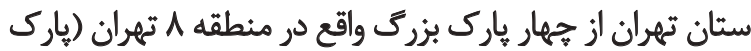

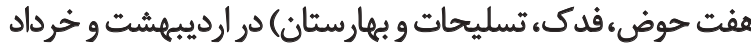

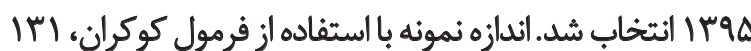

$$
\text { نفر تعيين شد. }
$$

استادان دانشكده روانشناسى و علومثربيتي داثشكاه علامه

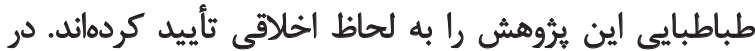

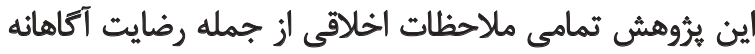

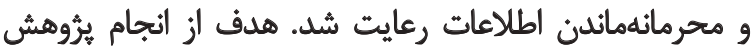




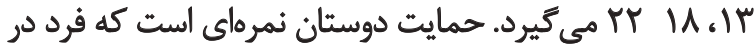

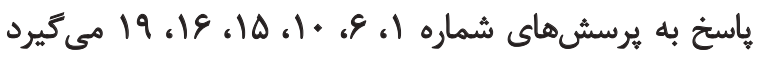

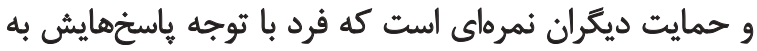

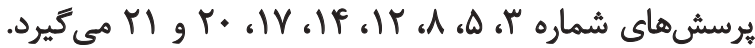

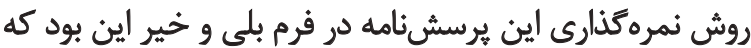

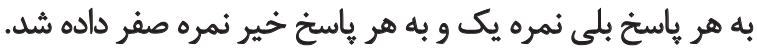

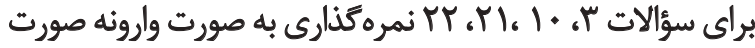

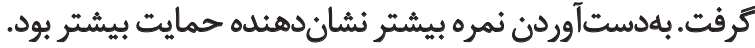

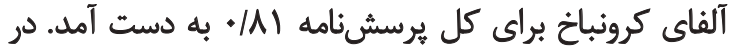

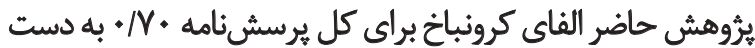

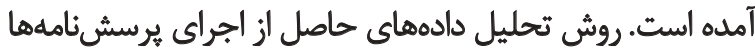

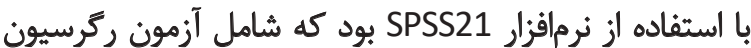
جندمتغيره همزمان و ساده مى نومارد.

يافتهها

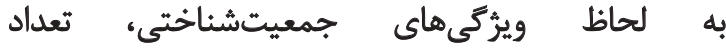

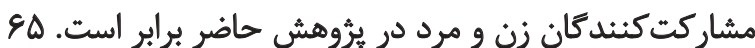

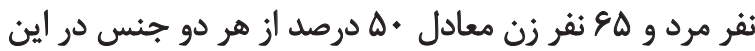

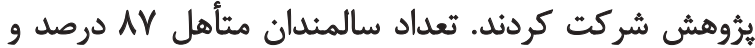

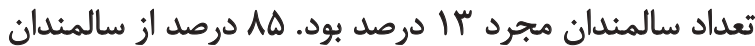

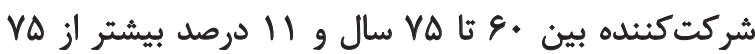

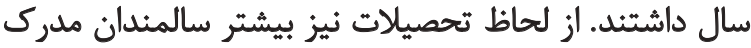

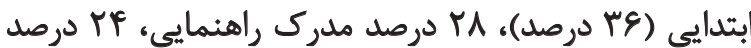

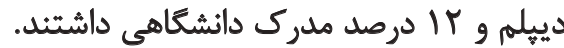

براي ياسخ مويى به اين فرض كه هاتوان مديريت استرس و

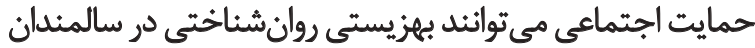

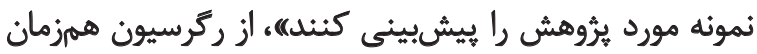

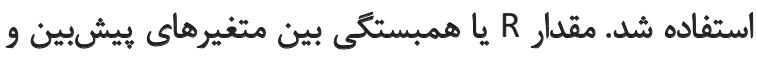

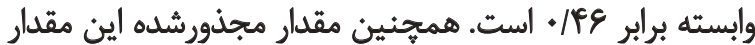

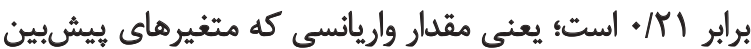

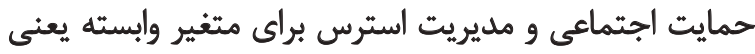

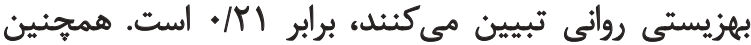

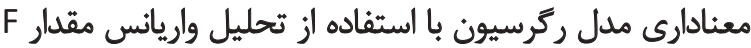

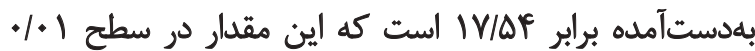

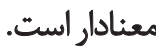

جدول شماره | ضرايب ركرسيونى مربوط به متغيرهاى

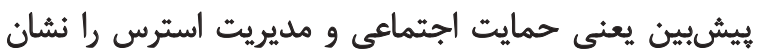

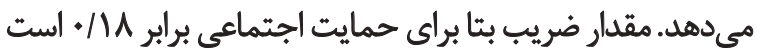

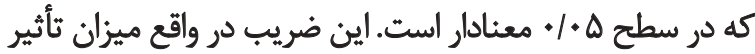

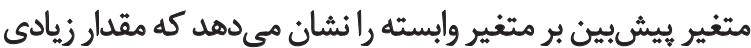

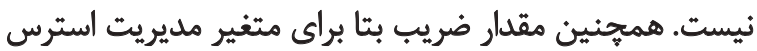

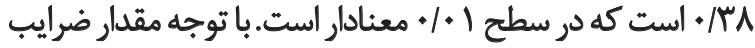
بتا براى دو متغير بيشبين، مى توان تفت متغير مديريت استرس

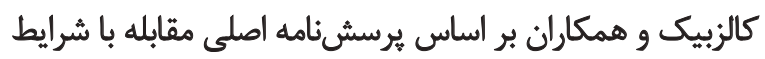

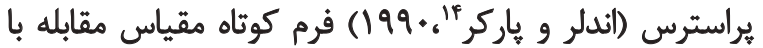

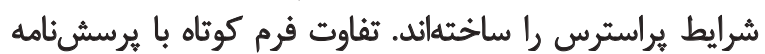

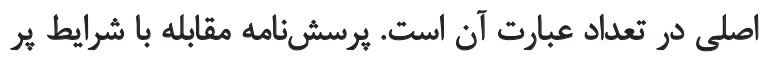

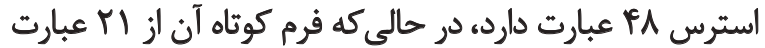

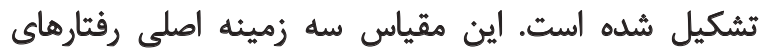

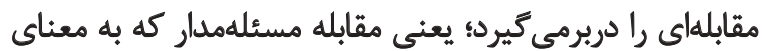

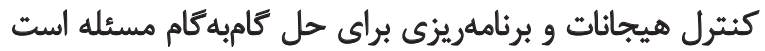

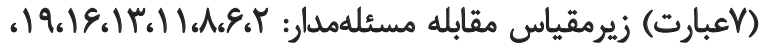

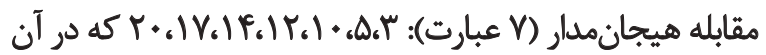

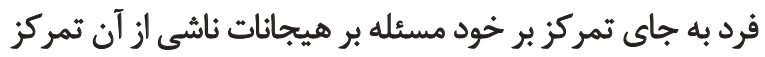

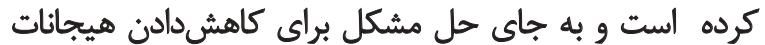

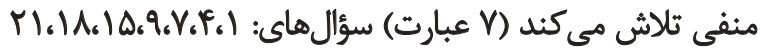

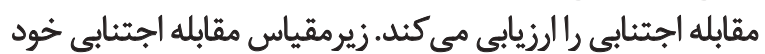

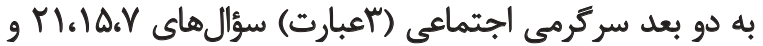

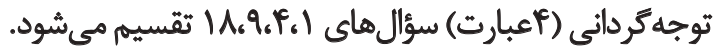
با توجه به اينكه نمرهكذارى به صورت بئج درجه ليكرت بودة،

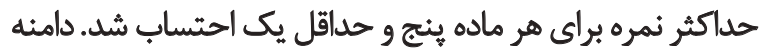

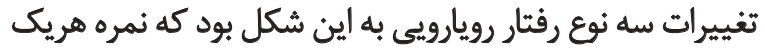

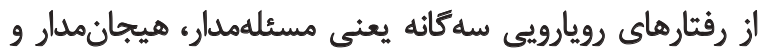

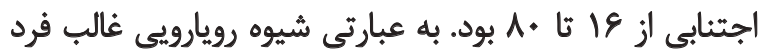

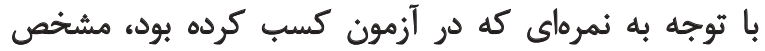

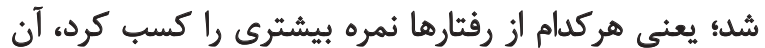

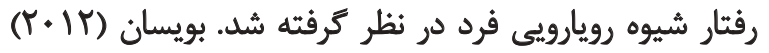

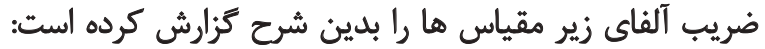

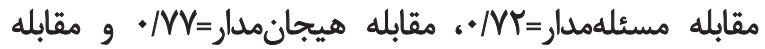

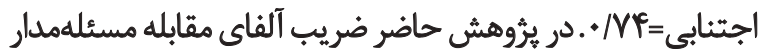

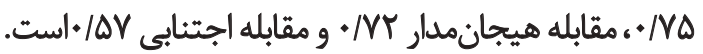

\section{مقياس سئجش حمايت الجتماعى فيليبي (SS-A)}

واكس، فيليبه، هلى، تامسون، ويليامز و استوارت 19 در سال

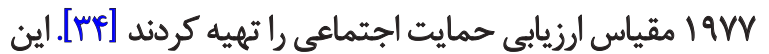

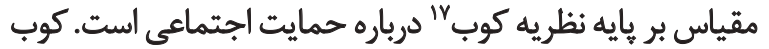

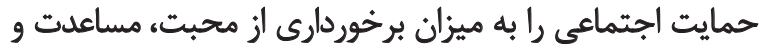

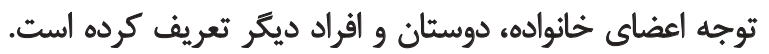

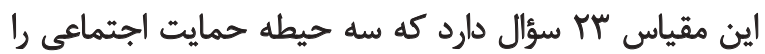

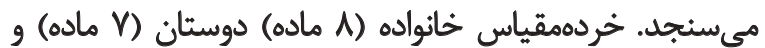

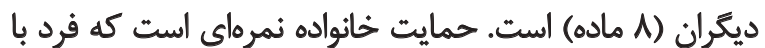

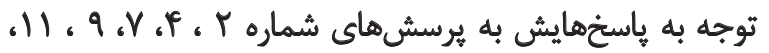

\section{Endler \& Parker}

15. Social support appraisals

16. Vaux, Phillips, Holly, Thomson, Williams \& Stewart 17. Cobb 
جدول ا. جدول نتيجه برازش مدل رترسيونى

\begin{tabular}{|c|c|c|c|c|c|}
\hline \multirow{2}{*}{$\mathbf{P}$} & \multirow{2}{*}{$\mathrm{T}$} & \multirow{2}{*}{ ضرايب الستاندارود } & \multicolumn{2}{|c|}{ ضرايب غيراستاندارد } & \multirow{2}{*}{ مدل } \\
\hline & & & Std. Error & B & \\
\hline $.1 .+1$ & g/qfi & & $\Delta / \Delta r \Delta$ & PNFIQ & مقدار ثابت \\
\hline.$/ . r V$ & T/MY & $\cdot|M|$ &.$/ M r r$ & . $/ \Delta T$ & حمايت اجتماعى \\
\hline $.1 .+1$ & PMa & $\cdot / r M$ & .1 .10 &.$/ 4 \cdot 9$ & توان مديريت استرس \\
\hline
\end{tabular}

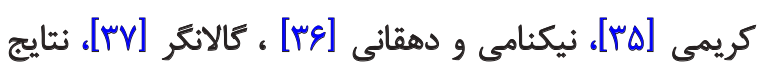

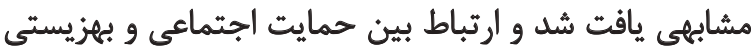

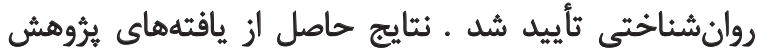

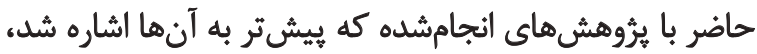

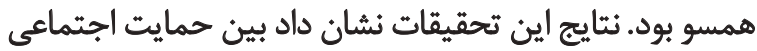
و بهزيستى روانشناختى رابطه وجود دارد.

در تبيين اين نتايج مىثنوان كفت حمايت اجتماعى

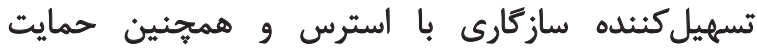

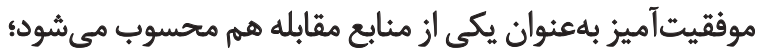

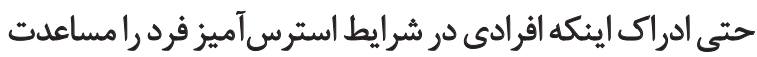

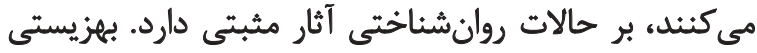

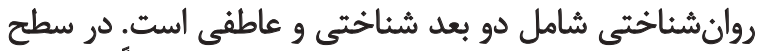

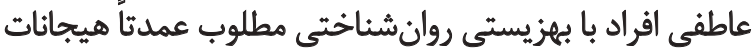

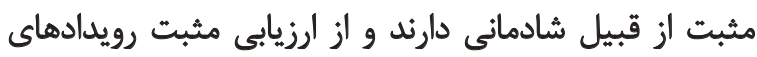

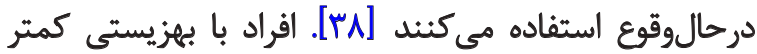

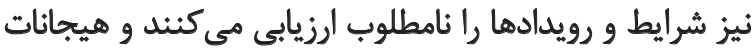

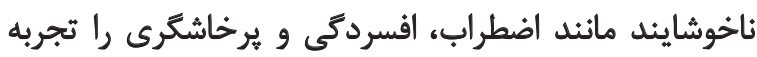

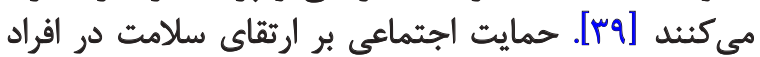

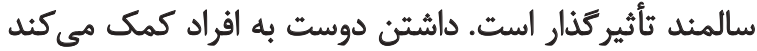

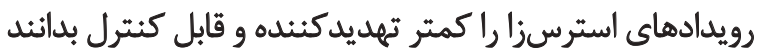

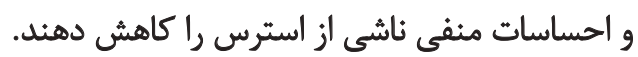

برم در مدل صميميت بيرامون حمايت اجتماعى بيان مى كند

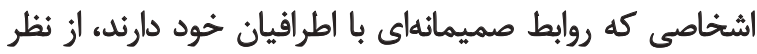

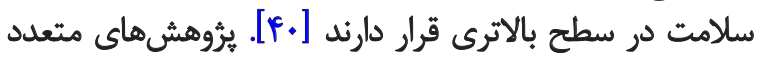

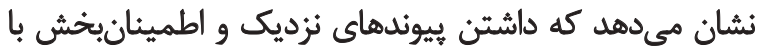

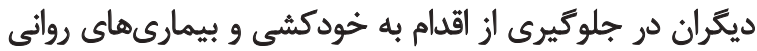

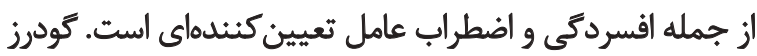

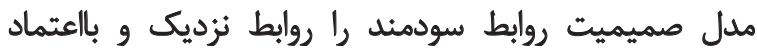

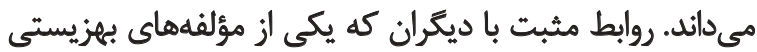

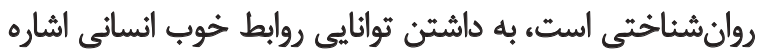

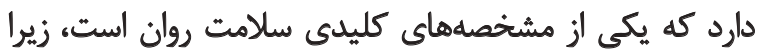

رابطه بيشترى با متغير وابسته، يعنى بجزيستى روانى دارد.

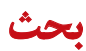

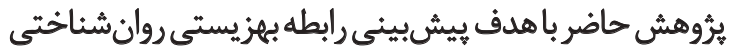

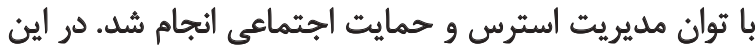

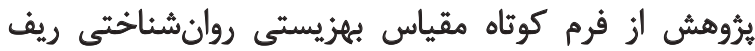

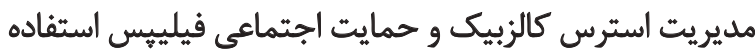

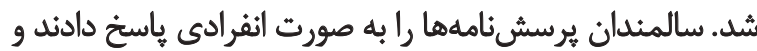

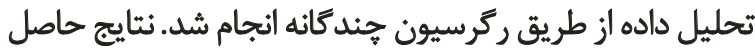

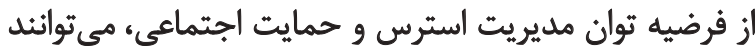

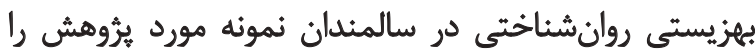

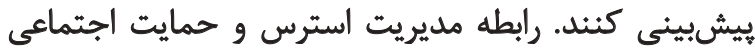

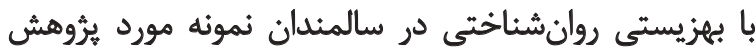

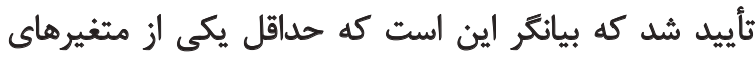

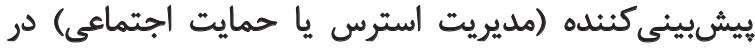

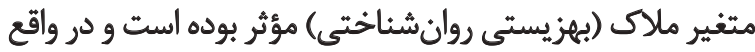

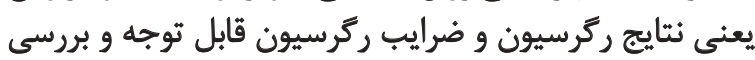

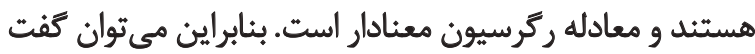

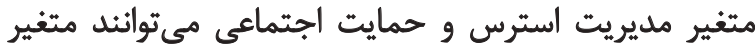

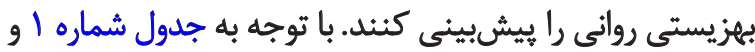

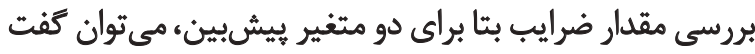

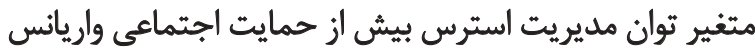

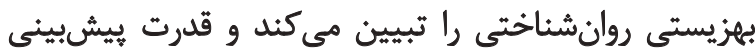
توان مديريت استرس بيشتر است.

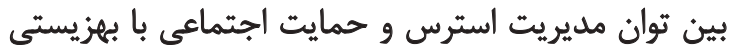

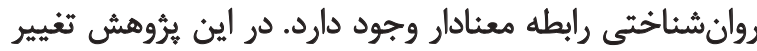

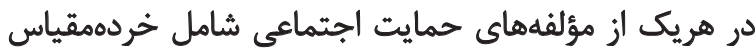

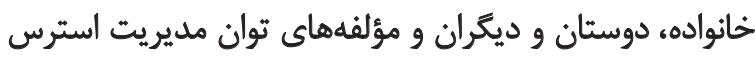

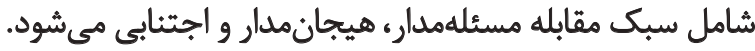

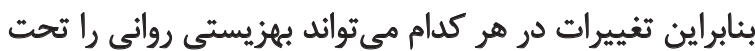

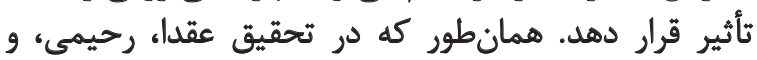


توان مديريت استرس و حمايت اجتماعى رابطه دارد و بيشينه

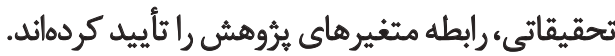

$$
\text { تشكر و قدرواني }
$$

در يايان از تمام كسانى كه در انجام اين يُوهش ما ما را يارى

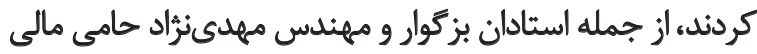
اين بُروهش، كمال تشكر و قدردانى را داريه.
آسيبشناسى معمولاً با اختلال در عملكرد اجتماعي مشخص

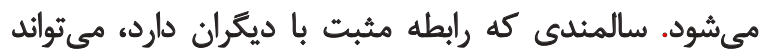

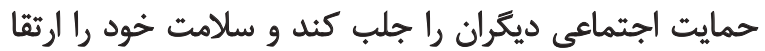

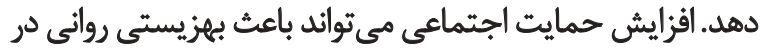

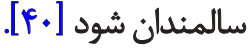

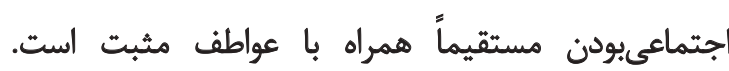

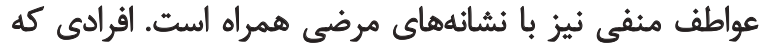

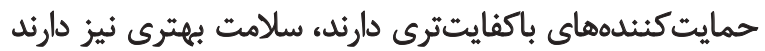

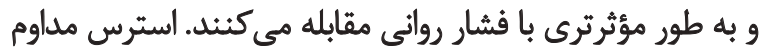

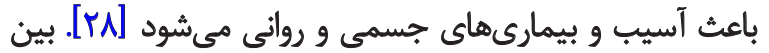

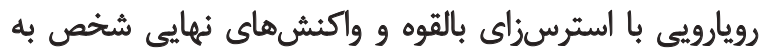

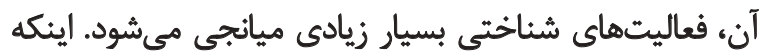

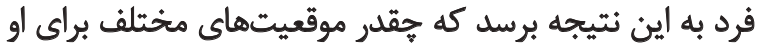

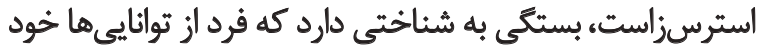

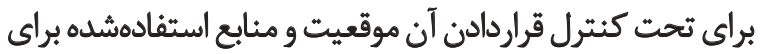

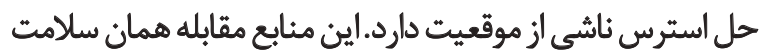

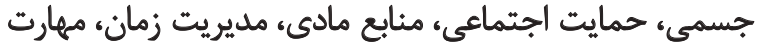

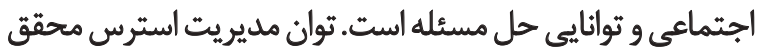

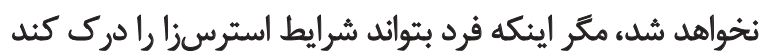

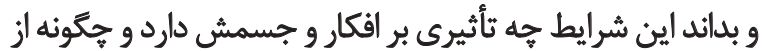

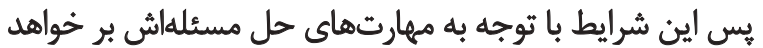

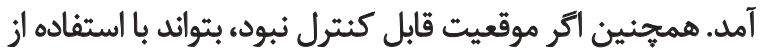

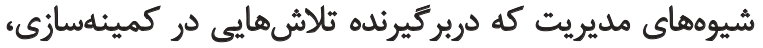

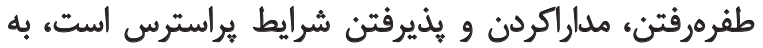
تعادل جسمى و روانى خود كمك كند.

با توجه به نتايج اين تحقيق و تحقيقات كذشتئه، به مسئولان،

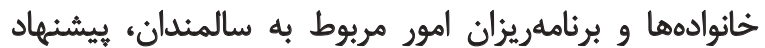

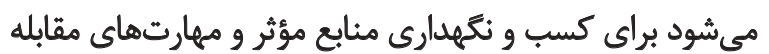

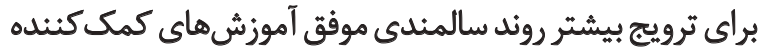

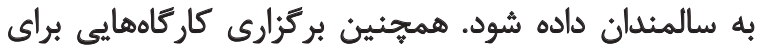

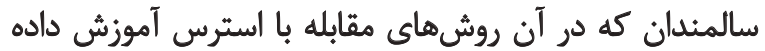

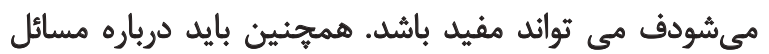

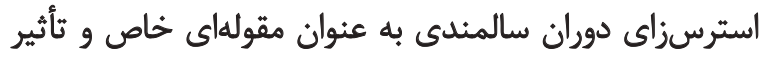

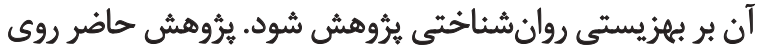

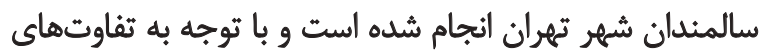

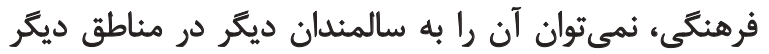

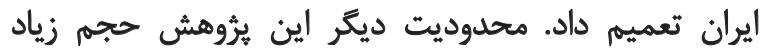

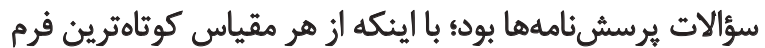

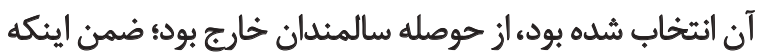
سطح بايين تحصيلات سالمندان نيز مزيد بر علت بود. تتيجه كيرى نهايى

نتايج يروهش حاضر نشان داد بهزيستى روانشناختى با 


\section{References}

[1] Kleink Chris L. Healthy People, Healthy Living [AM. Ghodarzi, Persian trans]. Tehran: Rasa; 2008.

[2] Saydshohadai M, Heshmat S, Seidfatemi N, Haghani H, Mehrdad N. [The spiritual health of seniors living in sanitarium and home residents (Persian)]. Iran Journal of Nursing. 2013; 26(81):11-20

[3] Brannon L, Feist J, Updegraff JA. Health Psychology: An Introduction to Behavior and Health. Belmont, California: Wadsworth Publishing; 2010

[4] Ryff CD. Psychological well-being revisited: Advances in the science and practice of eudaimonia. Psychotherapy and Psychosomatics 2014; 83(1):10-28. doi: 10.1159/000353263

[5] Myers DG, Diener E. Who is happy? Psychological Science. 1995; 6(1):10-9. doi: 10.1111/j.1467-9280.1995.tb00298.x

[6] Moghadami Poor M. [Stress management: The essential guide to thinking and working smarter (Persian)]. Tehran: Mehraban; 2007.

[7] Boshkani L. [Examination of correlation between social support, depression and anxiety in the seniors of Ahrom city (Persian)]. Paper presented at: The Iranian Conference on Psychology \& Behavioral Sciences. 22, January, 2015; Tehran, Iran.

[8] Renee Matich J, Sims LS. A comparison of social support variables between women who intend to breast or bottle feed. Social Science \& Medicine. 1992; 34(8):919-27. doi: 10.1016/02779536(92)90260-w

[9] Greenberg JS. Comprehensive Stress Management [M. Dehghani, B. Najariyan, Persian Trans]. Tehran: Roshd; 2008.

[10] Farshad M, Kalantar M, Farahbakhsh K. [The role of psychological well-being of family mental health promotion (Persian)] Paper presented at: The $2^{\text {nd }}$ National Conference on Sustainable Development in Educational Sciences and Psychology, Social and Cultural Studies. 5 June 2015; Tehran, Iran.

[11] Miller G, Chen E, Cole SW. Health psychology: Developing biologically plausible models linking the social world and physical health. Annual Review of Psychology. 2009; 60(1):501-24. doi: 10.1146/annurev.psych.60.110707.163551

[12] Stanton AL, Revenson TA, Tennen H. Health psychology: Psychological adjustment to chronic disease. Annual Review of Psychology. 2007; 58(1):565-92. doi: 10.1146/annurev. psych.58.110405.085615

[13] Trull TJ, Prinstein MJ. Clinical Psychology [M. Phirozbakht, Persian trans]. Tehran: Roshd; 2015.

[14] Darbonne A, Uchino BN, Ong AD. What mediates links between age and well-being? A test of social support and interpersonal conflict as potential interpersonal pathways. Journal of Happiness Studies. 2012; 14(3):951-63. doi: 10.1007/s10902012-9363-1

[15] Latten JJ. Life-course and satisfaction, equal for every-one? Social Indicators Research. 1989; 21(6):599-610. doi: 10.1007/ bf02217995

[16] Levenson RW. Expressive, physiological, and subjective changes in emotion across adulthood. In: Abeles N, Qualls $\mathrm{SH}$, editors. Psychology and the Aging Revolution: How We
Adapt to Longer Life. Washington, D.C.: American Psychological Association; 2000.

[17] Magai C. Emotions over the life span. In: Birren JE, Schaie KW, editor. Handbook of the Psychology of Aging. San Diego: Academic Press; 2001

[18] Diener E, Chan MY. Happy people live longer: Subjective well-being contributes to health and longevity. Applied Psychology: Health and Well-Being. 2011; 3(1):1-43. doi 10.1111/j.1758-0854.2010.01045.x

[19] Rowe JW, Kahn RL. Successful aging. New York: Pantheon/Random House; 1998

[20] Fingerman KL, Charles ST. It takes two to tango. Why older people have the best relationships. Current Directions in Psychological Science. 2010; 19(3):172-6. doi 10.1177/0963721410370297

[21] Thoits PA. Mechanisms linking social ties and support to physical and mental health. Journal of Health and Social Behaviour. 2011; 52(2):145-61. doi: 10.1177/0022146510395592

[22] Gow AJ, Corley J, Starr JM, Deary IJ. Which social network or support factors are associated with cognitive abilities in old age? Gerontology. 2013; 59(5):454-63. doi: 10.1159/000351265

[23] Alipoor F, Sajadi H, Forouzan A, Biglarian A. [The role of social support in elderly quality of life (Persian)]. Social Welfare. 2009, 9(33): 147-65.

[24] Motamedi Shalamzari A, Ezhehei J, Azad Falah P, Kiamanesh AR. [The role of social support on life satisfaction general well-being, and sense of loneliness among the elderly (Persian)]. Journal of Psychology. 2002; 6(2):115-33.

[25] Esgalhado G, Reis M, Pereira H, Afonso RM. [Influence of social support on the psychological well-being and mental health of older adults living in assisted-living residences (Portuguese)]. International Journal of Developmental and Educational Psychology. 2010; 1(1):267-78

[26] Chen Y, Feeley TH. Social support, social strain, loneliness, and well-being among older adults. Journal of Social and Personal Relationships. 2013; 31(2):141-61. doi $10.1177 / 0265407513488728$

[27] House JS. Work Stress and social Support (Addison-Wesley series on occupational stress). Glenview, Illinois: AddisonWesley Educational Publishers Inc; 1981.

[28] Sarafino EP. Health Psychology: Biopsychosocial Interactions [F. Shafee, E. Mirzaee, Persian trans]. Tehran: Roshd; 2012

[29] Shahbazi S, Heydari M, Shaykhi R. [The effect of problem solving course on students stress intolerance (Persian)] Journal of Shahrekord University of Medical Sciences. 2011 13(2):32-7.

[30] Tovel H, Carmel S. Maintaining successful aging: The role of coping patterns and resources. Journal of Happiness Studies. 2013; 15(2):255-70. doi: 10.1007/s10902-013-9420-4

[31] Trouillet R, Doan-Van-Hay LM, Launay M, Martin S. Impact of age, and cognitive and coping resources on coping Canadian Journal on Aging. 2011; 30(4):541-50. doi: 10.1017/ s0714980811000456 
[32] Khanjani M, Shahidi S, Fath-Abadi J. [Factor structure and psychometric properties of the Ryff's scale of Psychological well-being, short form (18-item) among male and female students (Persian)]. Journal of Thought \& Behavior in Clinical Psychology .2014; 8(32):27-36.

[33] Calsbeek H, Mieke R, Henegouwen GP, Dekker J. 5 factor structure of the coping inventory for stressful situation (CISS21) in adolescents and young adults with chronic degestive disorders. In: Calsbeek H, editor. The Social Position of Adolescents and Young Adults With Chronic Digestive Disorders. Utrecht: NIVEL; 2003.

[34] Vaux A, Phillips J, Holly L, Thomson B, Williams D, Stewart D. The Social Support Appraisals (SS-A) scale: Studies of reliability and validity. American Journal of Community Psychology. 1986; 14(2):195-218. doi: 10.1007/bf00911821

[35] Aqda N, Rahimi FM, Karimi A, Vazitan M. [Comparative study of mental well-being and quality of life among the elderly in Yazd (Persian)]. Paper presented at: The International Conference on Humanities and Behavioral Studies. 17 October 2014; Tehran, Iran.

[36] Niknami M, Dehghani F, Bouraki SH, Kazemnejad L, Soleimani R. [Strategies among students of Guilan University of Medical Sciences (Persian)]. Journal of Holistic Nursing And Midwifery. 2014; 24(4):62-68.

[37] Gallagher LP, Truglio-Londrigan M. Community support: Older Adults' perceptions. Clinical Nursing Research [Internet]. SAGE Publications; 2004; 13(1):3-23. doi: $10.1177 / 1054773803259466$

[38] HakimiTehrani L, Fatehizadeh I, Baghban I. [The effects of emotional safety training on marital conflicts and subjective well-being of married women in Isfahan (Persian)]. Journal of Women \& Society. 2012; 3(3):105-124.

[39] Shahsiah M, Bahrami F, Mohebi S, Tabarrai Y. [Correlation between mental well-being and marital life quality of couples (Persian)]. Qom University of Medical Sciences Journal. 2012; 5(4):61-7.

[40] Goudarz M, Foroughan M, Makarem A, Rashedi V. [Relationship between social support and subjective well-being in older adults. (Persian)]. Iranian Journal of Aging. 2015; 10(3):110-19. 